\title{
Transmission Electron Microscopy of Ultramicrotome Samples of Porous InSb formed by Ion Implantation
}

\author{
D. J. Llewellyn, S. M. Kluth, and M. C. Ridgway
}

Department of Electronic Materials Engineering, Research School of Physical Sciences and Engineering, Australian National University, Canberra, ACT 0200, Australia

InSb is a narrow band gap semiconductor with a high electron mobility used in magnetoresistive sensors, hall elements, and for astronomy and military applications [1]. Ion implantation, commonly used in device fabrication, can cause InSb to become porous [2-4]. In this work we look at methods used to examine the porous microstructure of ion irradiated InSb.

Single crystalline InSb wafers were implanted at room temperature (RT) with $1 \mathrm{MeV},{ }^{69} \mathrm{Ga}^{+}$ions . Scanning electron microscopy (SEM) images were obtained (from the cross sectioned cleaved wafer) for two different implant fluences $\left(2.5 \times 10^{14} \mathrm{at}_{\mathrm{cm}}{ }^{-2}\right.$ and $\left.1 \times 10^{15} \mathrm{at} . \mathrm{cm}^{-2}\right)$ these are shown in Fig. 1 (a-c) and (d-f), respectively. Panels (a) and (d) show the whole of the implanted region. For the lower fluence the porous layer is $\sim 1.5 \mu \mathrm{m}$ thick. Increasing the implant fluence by a factor of four causes the porous region to expand almost fourfold to $5.7 \mu \mathrm{m}$. Higher magnification images of the surface region are displayed in panels (b) and (e). For the $2.5 \times 10^{14} \mathrm{at}_{\mathrm{cm}}^{-2}$ implant the surface

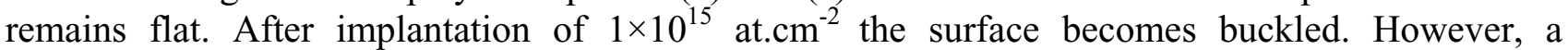
continuous surface layer approximately $20 \mathrm{~nm}$ thick can still be observed. At $2.5 \times 10^{14}$ at.cm ${ }^{-2}$, large voids give the material the sponge-like texture reported by Destefanis and Gailliard [2] (panel c). For the $1 \times 10^{15}$ at. $\mathrm{cm}^{-2}$ implant, the microstructure changes dramatically resulting in a network of straight rods approximately $20 \mathrm{~nm}$ in diameter (panel f).

To analyse these structures with transmission electron microscopy (TEM) several methods have been employed. The simplest was to deposit mechanically removed porous material onto carbon grids. This, however, negates any depth dependent information. Traditional cross-sections could be produced for the less porous material, however, this was not feasible with the highly porous structure of Fig.1(d). To provide stability for the porous material it was infiltrated under pressure, (after evacuation) with a vacuum compatible epoxy resin then sectioned using a Reichert Ultracut Microtome and Diatome diamond knife. Sections were cut at about 70nm and mounted on 200 mesh holey carbon support grids.

The entire porous layer is visible in the TEM image of Fig. 2. The rod-like structures in the upper region are no longer interconnected due to the minimal thickness of the ultramicrotome section. The lower interface demonstrates the evolution of the microstructure as a function of implant fluence due reduction in the damage deposition at these depths arising from the Gaussian implant profile. Below the porous structure the dark region contains spherical voids as small as $3 \mathrm{~nm}$ and dislocation loops. Diffraction patterns indicate the material is partly polycrystalline. At lesser depths, the voids increase in size, remaining more or less spherical until they exceed $\sim 20 \mathrm{~nm}$. Above this, the voids become elongated in the direction normal to the surface and the structure starts to open up. The large voids transform into the plate and rod-like structures observed with SEM. 
In summary, our method of resin infiltration followed by microtoming enables TEM characterisation of porous layers when conventional thinning and ion-beam milling fails.

\section{References}

[1] R. Szweda, III-Vs Review 17 (2004) 32.

[2] G.L. Destefanis and J.P. Gailliard, Appl. Phys. Lett. 36 (1980) 40.

[3] G.L. Destefanis et al., Nucl. Instr. Meth. B 182/183 (1981) 637.

[4] M. Shaanan, R. Kalish and V. Richter, Nucl. Instr. Meth. B 7/8 (1985) 443.

The aid of Lily Shen is gratefully acknowledged.
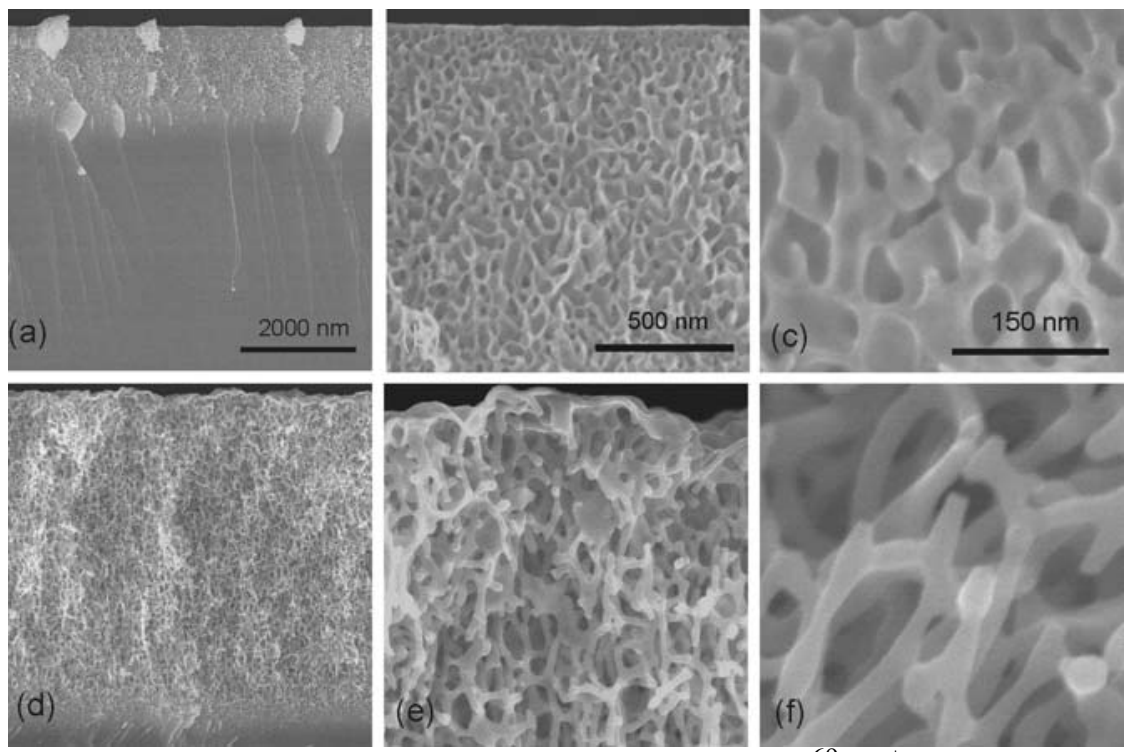

FIG. 1. Cross-sectional SEM images of $1 \mathrm{MeV}^{69} \mathrm{Ga}^{+}$implants into InSb (a-c) $2.5 \times 10^{14}$ at.cm ${ }^{-2}$, (d-f) $1 \times 10^{15} \mathrm{at}^{-\mathrm{cm}^{-2}}$. (The scale bars apply to both images in the same column.) (a) and (d) show the entire porous layer, (b) and (e) are magnifications of the surface region, and (c) and (f) show the microstructure at center of the porous region.

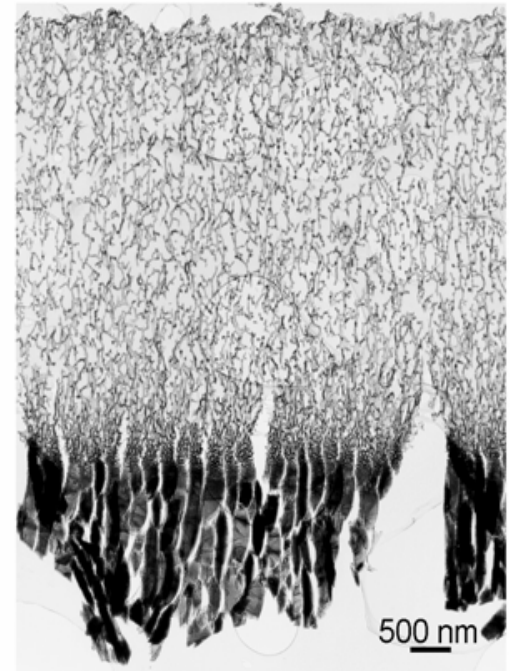

FIG. 2. TEM image of $1 \times 10^{15}$ at. $\mathrm{cm}^{-2}, 1 \mathrm{MeV}$, ${ }^{69} \mathrm{Ga}^{+}$implants into InSb. The porous material has been infiltrated with resin and sectioned using a diamond ultramicrotome. 